\title{
A 32-mW 320-MHz Continuous-Time Complex Delta-Sigma ADC for Multi-Mode Wireless-LAN Receivers
}

Jesus Arias, Member, IEEE, Peter Kiss, Member, IEEE, Vladimir Prodanov, Vito Boccuzzi, Mihai Banu, Fellow, IEEE, David Bisbal, Jacinto San Pablo, Luis Quintanilla, and Juan Barbolla

\begin{abstract}
We present an experimental continuous-time complex delta-sigma multi-bit modulator, implemented in standard 0.25- $\mu \mathrm{m}$ CMOS technology and meeting all major requirements for application in IEEE $802.11 \mathrm{a} / \mathrm{b} / \mathrm{g}$ wireless $\mathrm{LAN}$ receivers. The clock frequency is $320 \mathrm{MHz}$, producing an oversampling ratio of 16 for 20 MHz channel bandwidths. The modulator supports two operation modes for zero-IF and low-IF receiver architectures respectively, requires a single $2.5-\mathrm{V}$ power supply, and dissipates only $32 \mathrm{~mW}$ of power. The measured peak signal-to-noise ratio is 55 dB. Further experimental results using sine-wave and OFDM test signals are also presented.
\end{abstract}

Index Terms-Analog-to-digital conversion, sigma-delta modulation, wireless LAN.

\section{INTRODUCTION}

W IRELESS-LANs based on the IEEE 802.11 standard achieve high data rates through the use of wide channel bandwidths and efficient modulation techniques such as CCK and OFDM (802.11a/b/g standards). The economic drive for low cost and low power mandates the use of highly integrated transceivers based on zero-IF (ZIF) or low-IF (LIF) architectures. The former are well suited for $802.11 \mathrm{~b}$ applications since the CCK modulation allows AC baseband coupling, thus eliminating any signal-related DC offsets. For 802.11a/g OFDM applications where baseband $\mathrm{AC}$ coupling is very difficult, LIF receivers at $10 \mathrm{MHz}$ are better suited, especially since the adjacent channel rejection specifications are relaxed. Therefore, in multi-mode $802.11 \mathrm{a} / \mathrm{b} / \mathrm{g}$ applications, it is beneficial to use a single ADC block to digitize either an I/Q-pair of real signals for CCK or a single 10-MHz-centered LIF signal for OFDM.

The high dynamic range of $802.11 \mathrm{a} / \mathrm{g}$ OFDM enforces fundamental limitations on the minimum power dissipation achievable. For example, a substantial power penalty is paid when using typical Nyquist-rate ADCs preceded by high-order, high dynamic range fully integrated channel filters. A better design for low power would be to employ over-sampled A/D conversion preceded by low-order channel filters [1]. Furthermore, this approach would yield superior I/Q-balance properties over the

Manuscript received November 6, 2004; revised October 3, 2005.

J. Arias, D. Bisbal, J. San Pablo, L. Quintanilla, and J. Barbolla are with the Dpto. de E. y Electrónica, E.T.S.I. Telecomunicación, Universidad de Valladolid, Valladolid, Spain.

P. Kiss and V. Prodanov are with Agere Systems, Allentown, PA 18109 USA.

V. Boccuzzi is with Analog Devices, Somerset, NJ 08873 USA.

M. Banu is with MHI Consulting, LLC, Murray Hill, NJ 07974 USA.

Digital Object Identifier 10.1109/JSSC.2005.862346 channel bandwidth. In order to prove the feasibility of such an attractive architecture, it is necessary to first demonstrate a low-power $\Delta \Sigma \mathrm{ADC}$ meeting the 802.11 requirements.

Single-bit $\Delta \Sigma$ ADCs are widely used in low-speed applications, such as audio, where a very high oversampling ratio (OSR) with respect to the input signal bandwidth is easily attainable. In the case of $802.11 \mathrm{a} / \mathrm{g}$ wireless-LAN receivers where the channel bandwidth is $20 \mathrm{MHz}$, a similar frequency-scaled design would result in gigahertz clock frequencies. This operating speed is too fast and not practical for currently available CMOS technology. In addition, the classical $\Delta \Sigma$ ADCs are built as discrete-time circuits with switched-capacitor integrators. Discrete-time circuits require operational amplifiers with gain-bandwidth products about 2 to 10 times the clock frequency (depending on the closed-loop gain of each particular integrator) which would lead to high power consumption and noise.

In order to realize a practical $\Delta \Sigma$ ADC for 802.11, two changes are applied with respect to conventional topologies. First, a multi-bit quantizer is used instead of the widely popular single-bit design. This achieves a reasonably high signal-to-noise ratio (SNR) with a low OSR [1]. The alternative possibility for increased SNR, i.e., employing a high-order loop filter, would result in potential stability problems and high sensitivity to coefficient variations. The inherent DAC nonlinear characteristic, which is the main drawback of multi-bit quantizers, can be compensated using dynamic element matching techniques [2], [3]. Second, the loop-filter of the ADC is implemented as a continuous-time (CT) circuit. In this way, the bandwidth and power requirements for this block are minimized even at high sampling clock frequencies [4]-[6].

Recently, CT $\Delta \Sigma$ ADC designs in CMOS have grown in popularity. Their low-power potential for medium/high (10-15 bits) resolution and narrow bandwidth $(1-2 \mathrm{MHz}$ ) has been proven by several successful designs [7]-[10]. Furthermore, recent CT $\Delta \Sigma$ ADCs have also targeted wideband (10-20 MHz) 10-13-bit resolution applications [11]-[13]. These are attractive since equivalent switched-capacitor implementations pose great challenges on the circuit design and require power-hungry opamps [14].

The ZIF and LIF receivers perform RF to IF conversion by generating an in-phase IF component called $I$ and a quadrature IF component called $Q$. These components, which are necessary to carry the desired channel information and remove the RF image (image rejection) can be mathematically represented 

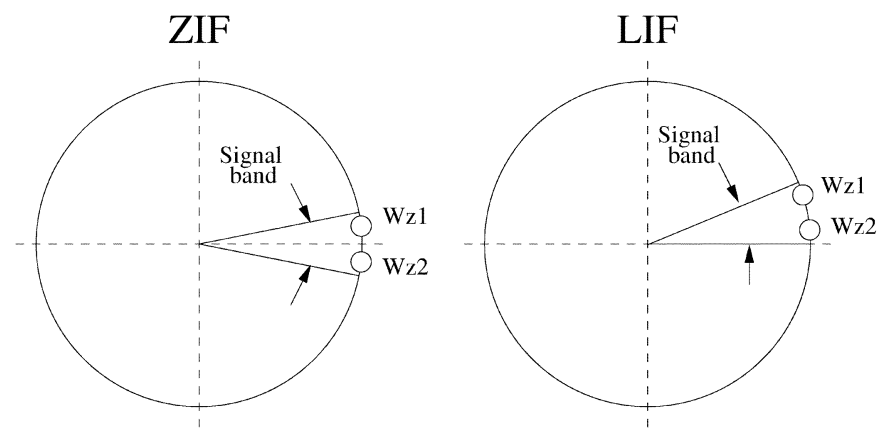

Fig. 1. Location of the NTF zeros in the Z-domain for the ZIF and LIF modes. The signal bandwidth is $20 \mathrm{MHz}$.

as the real and imaginary parts of a complex IF signal. The digitization of this complex IF signal requires a complex ADC, which in the case of ZIF may be just a pair of two real conventional ADCs. However, for LIF the use of two real ADCs would double the clock frequency and the digitizing bandwidth. This must be done to capture the IF signal but it wastes power since it is not necessary to digitize the negative frequencies where the adjacent channel resides and there is no desired channel energy. A complex $\Delta \Sigma$ ADC with a signal band that includes only the positive frequencies can be designed using two conventional $\Delta \Sigma$ ADCs clocked like in the ZIF case complemented with proper cross-coupling elements [15]. This topology yields a complex noise transfer function (NTF) shifted toward the positive frequencies, centered at $10 \mathrm{MHz}$ and having a bandwidth of $20 \mathrm{MHz}$. The power dissipation of this complex ADC is not substantially higher than that of a dual real ADC for ZIF because the cross-coupling elements have a small contribution to the total power budget.

In this paper, we report the first complex, continuous-time $\Delta \Sigma$ ADC designed for IEEE $802.11 \mathrm{a} / \mathrm{b} / \mathrm{g}$ wireless LANs. The proposed complex CT $\Delta \Sigma$ ADC has the lowest power consumption (32 $\mathrm{mW}$ for complex operation, $15 \mathrm{~mW}$ for single real ADC) and smallest active chip area $\left(0.44 \mathrm{~mm}^{2}\right)$ within the medium resolution (10 bit) and wide bandwidth $(10-20 \mathrm{MHz})$ state-of-the art $\Delta \Sigma$ ADCs [11]-[14]. The low power consumption is achieved by choosing a simple second-order 3-bit modulator, by employing low-noise and high-linearity $\mathrm{Gm}$ - $\mathrm{C}$ integrators in the loop filter, and by judicious analog design.

The structure of the modulator and the circuit implementation are shown in Sections II and III, respectively. Experimental results, using both sine-wave and OFDM inputs, are presented and discussed in Section IV. Conclusions are summarized in Section $\mathrm{V}$.

\section{COMPleX $\Delta \Sigma$ Modulator DesigN}

\section{A. Architecture Selection}

The design's peak SNR target was set to $60 \mathrm{~dB}$, to allow $5 \mathrm{~dB}$ margin above the required $55 \mathrm{~dB}$. In order to achieve this SNR with an OSR of only 16 , a second-order, multi-bit $\Delta \Sigma$ modulator with a 3-bit quantizer and zero-optimized NTF [16] was chosen. We started with a NTF for a discrete-time $\Delta \Sigma$ modulator and mapped it to a CT modulator. In Fig. 1, the location of the zeros of the discrete-time modulator NTF is shown for both (a)

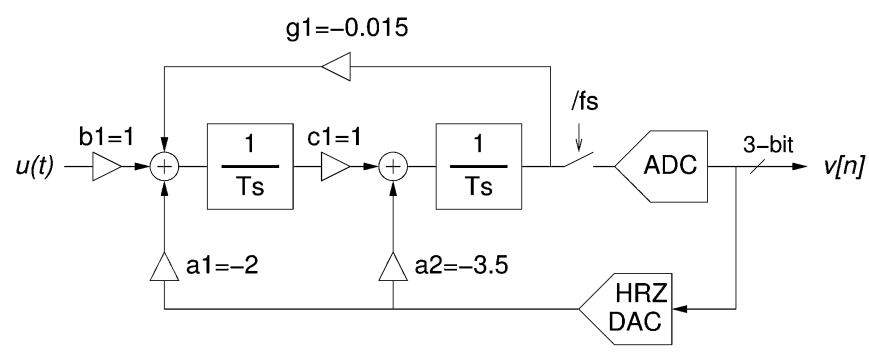

(b)

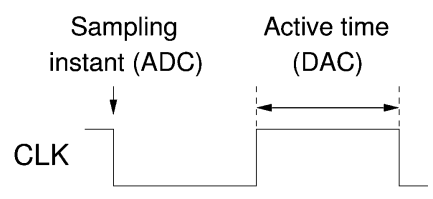

Fig. 2. (a) Diagram of the second-order, continuous-time, multibit, real modulator and (b) Timing.

the zero-IF mode (ZIF mode) and low-IF mode (LIF mode). In both cases, the NTF can be written as

$$
\operatorname{NTF}(z)=z^{-2}\left(z-W_{Z 1}\right) \cdot\left(z-W_{Z 2}\right)
$$

where $W_{Z 1}$ and $W_{Z 2}$ are the zeros for the selected mode (ZIF or LIF).

As mentioned before, the ZIF mode is intended for direct-conversion receivers, where the signal band is centered at DC. Therefore, the two NTF zeros are complex conjugate, and then the modulator can be built with two isolated real modulators, each having the following NTF:

$$
\operatorname{NTF}(z)=1-1.98792 z^{-1}+z^{-2} .
$$

In the LIF mode, the NTF shows a frequency shift toward positive frequencies $(+10 \mathrm{MHz})$. The two zeros are no longer complex conjugate, and the resulting NTF has complex coefficients implemented as a coupling between the $I$ and $Q$ modulators. The corresponding zero values are

$$
\begin{aligned}
& W_{Z 1}=(0.9534+j 0.3015) \\
& W_{Z 2}=(0.9963+j 0.0863) .
\end{aligned}
$$

\section{B. CT Modulator Implementation}

The realization of the ZIF/LIF complex modulator began with the design of a real CT modulator, which would be used in a pair of two identical blocks for ZIF mode. The block diagram of this circuit is shown in Fig. 2(a). The coefficients were obtained by mapping the NTF of the CT modulator to (2). The modified Z-transform [17] was used taking into account the timing of the half-return-to-zero (HRZ) DAC waveform.

The ADC in Fig. 2(a) samples its input on the falling edge of the clock, but its output signal is not needed in the closed-loop system until the second half of the clock cycle due to the HRZ waveform [Fig. 2(b)]. In this way, the ADC has half a cycle to perform the analog-to-digital conversion. The effect of the ADC comparator metastability on modulator performance is reduced [6]. The DAC is turned on and off on each cycle providing a half-delayed, return-to-zero signal. Modulators using return-to- 


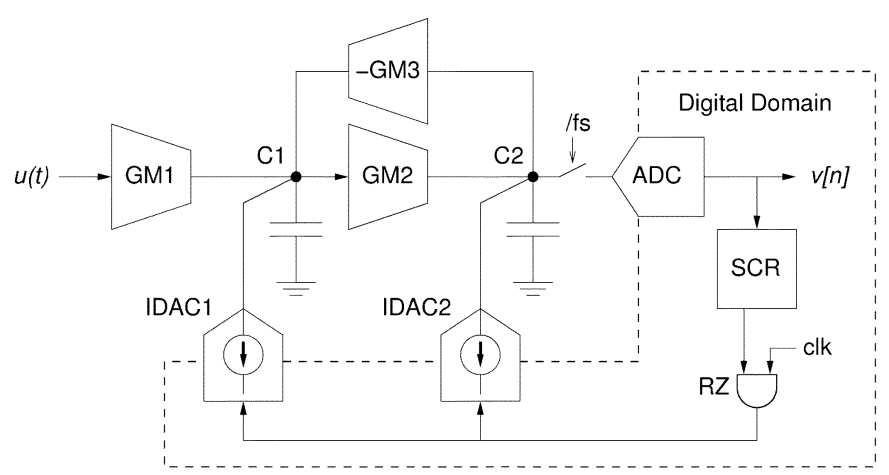

Fig. 3. Block diagram of a $G m-C$ implementation of the real modulator.

zero signals exhibit less performance degradation due to turnon/turn-off transients of the DAC because these transients do not depend on previous output data [4], [5]. However, they exhibit higher clock jitter sensitivity.

The coefficients shown in the modulator of Fig. 2(a) realize the correct NTF but do not provide optimum dynamic range. Scaling may be applied to adjust the amplitude distribution of the two integrators to proper values without changing the NTF. Such scaling was done taking into account the input range of the transconductors used in the integrators $( \pm 500 \mathrm{mV})$.

The CT integrators of Fig. 2(a) are implemented as transconductors loaded with capacitors, resulting in the schematic of Fig. 3. Note that while this schematic shows a single-ended circuit for clarity, the actual modulator was built using fully differential circuits.

As can be seen in Fig. 3, the sampling operation occurs only at the ADC input, while the loop filter is a CT circuit providing implicit aliasing rejection for this type of modulator [6]. The DAC of Fig. 2(a) is replaced by two current-output DACs, implemented as arrays of identical, switched current sources controlled by a thermometer-code digital input. Multi-bit modulators are sensitive to DAC nonlinearity. In order to reduce this effect, a scrambler, SCR, is added between the ADC output and the DAC inputs. This circuit improves the linearity of the DACs by selecting a different set of DAC elements on each sample, thus performing dynamic element matching. A data weighted averaging algorithm (DWA) [2] was chosen for the scrambler due to its simplicity and because system level simulations show that this algorithm is good enough to remove the effects of DAC element mismatches as high as $2 \%$ or even more. Quadrature DEM algorithms have been proposed in order to improve the image rejection of complex $\Delta \Sigma$ ADCs [18], [19]. However, these algorithms cannot correct the mismatch between $I$ and $Q$ channels of the entire complex modulator. In this case, such mismatches can be as high as those of the DAC elements. In our design, a simpler, independent scrambler is used for each real modulator and image rejection accuracy is obtained through proper layout techniques. The layout of the elements in the $I$ and $Q$ channels, including the DAC elements, the capacitor units, the transconductors, and the resistors of the ADCs, were interlaced and laid out following a common-centroid topology.

Two real modulators can be combined into a single complex modulator as can be seen in Fig. 4. Without the cross-coupling

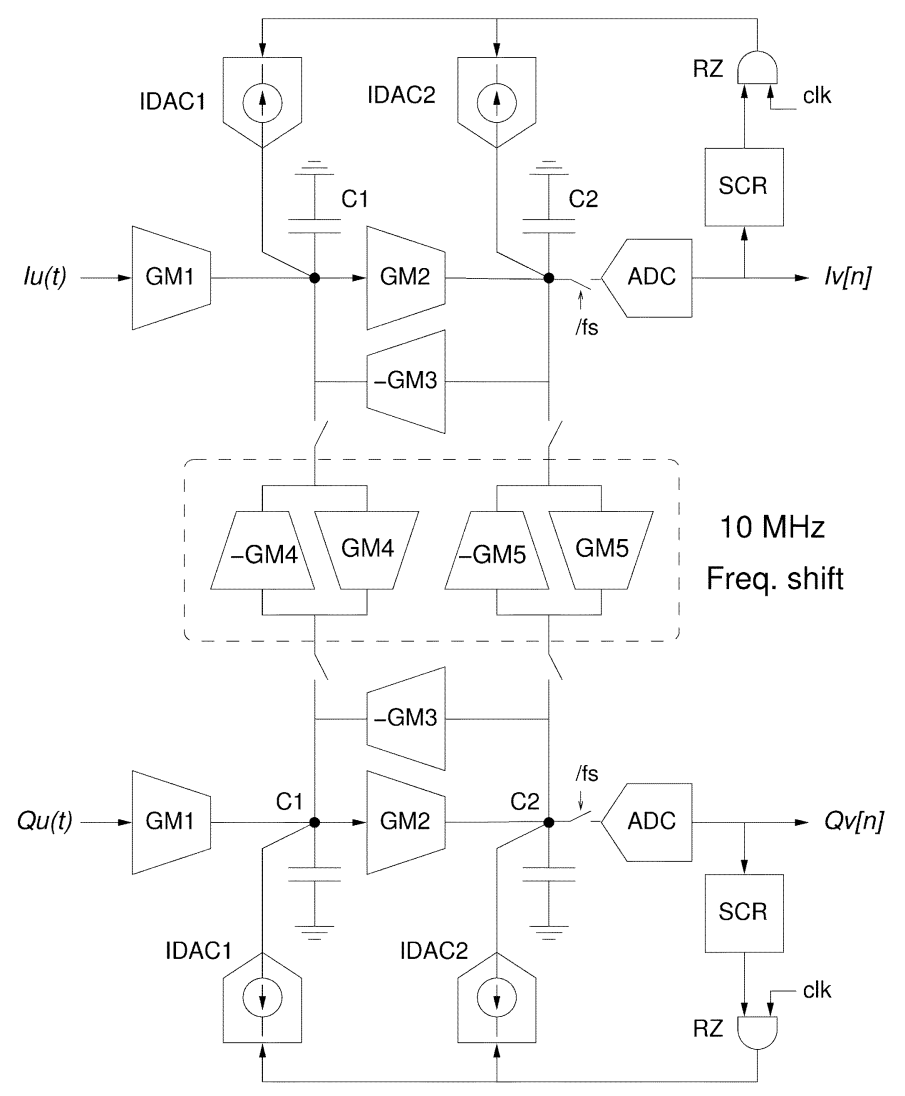

Fig. 4. Complex modulator schematic.

transconductors, each real modulator works independently and the resulting NTF of the complex modulator is centered around DC [Fig. 5(a), ZIF mode]. If transconductors $G M_{4}$ and $G M_{5}$ are enabled, a $+10 \mathrm{MHz}$ frequency shift is obtained [Fig. 5(b), LIF mode] . Therefore, by enabling the coupling transconductors we can change from a modulator for zero-IF receivers (ZIF mode) to a modulator for low-IF receivers (LIF mode). The optimum modulator mode can be selected dynamically in a multimode receiver based on this ADC.

\section{Impact of Nonideal Effects on Performance}

The modulator architecture was verified using a behavioral-level simulator, including nonideal effects such as nonlinear transconductors, integrators with finite DC gain and a nondominant pole, mismatches, thermal noise, and clock jitter. Some of these nonideal effects have been included in the simulated spectra of Fig. 5. The modulator showed low sensitivity to process variation, allowing for higher than $20 \%$ relative variations in the main modulator elements, namely, transconductors, capacitors, and DACs. The assumed relative mismatch was estimated at $1 \%$, resulting in no significant SNR loss when the scramblers are active, and in an approximately $35 \mathrm{~dB}$ image rejection. Experimental results show that this mismatch estimation was pessimistic (see Section IV).

CT modulators are known to be sensitive to clock jitter because the inaccuracies of the clock signal are translated into a charge error at the output of the DACs [6]. If the clock jitter is the only source of noise present, and if only the jitter of the first 
(a)

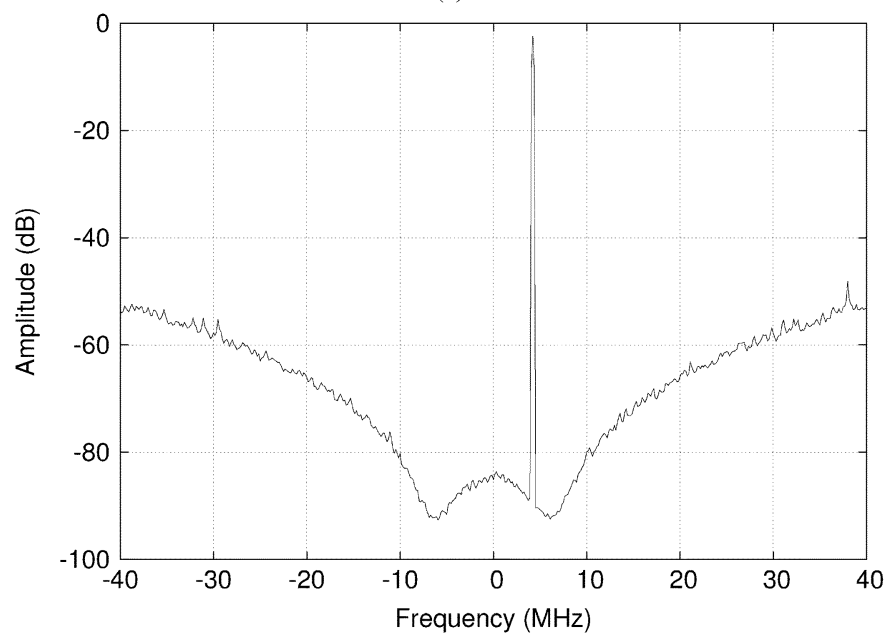

(b)

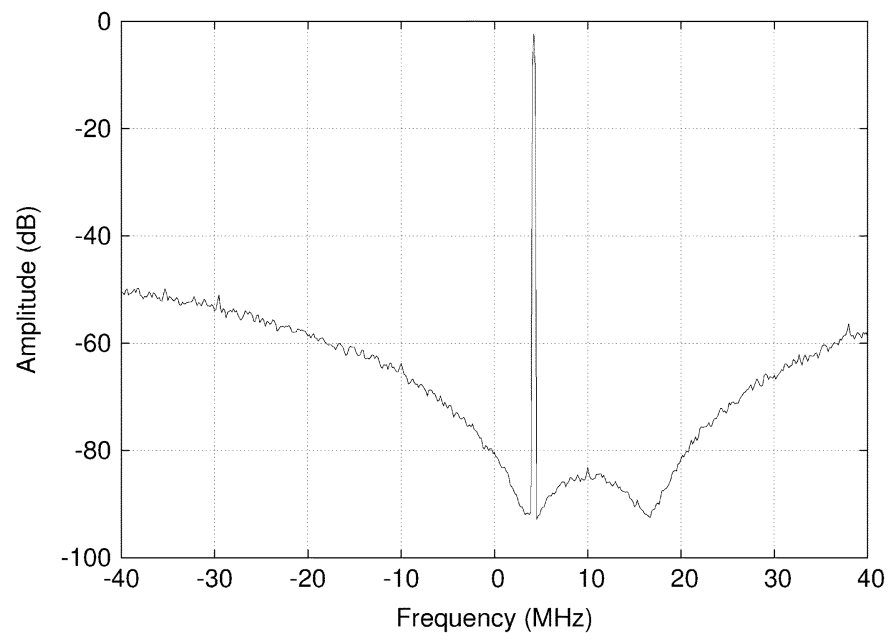

Fig. 5. Simulated spectra for (a) ZIF mode. (b) LIF mode. Simulations were done using a behavioral ADC model including finite DC gain, an integrator nondominant pole, and thermal noise. Clock jitter is not included in order to keep the NTF notches clearly visible. A 4.3 MHz complex test tone has been applied to the inputs.

DAC is considered (the following DAC noise is shaped and can be neglected), the expected SNR for the modulator of Fig. 2 is

$$
\operatorname{SNR}(d B)=10 \cdot \log _{10}\left(\frac{\mathrm{OSR}}{32 \frac{\sigma_{\mathrm{jitter}}^{2}}{T^{2}}}\right)
$$

where $\sigma_{\mathrm{jitter}}$ is the standard deviation of the clock period (jitter) and $T$ the nominal clock period (3.125 ns). Therefore, a clock signal with a jitter level better than $4 \mathrm{ps}$ is required $(\sigma / T<$ $0.12 \%)$ in order to avoid a significant performance degradation.

\section{CiRCUIT IMPLEMENTATION}

The modulator was implemented in a standard $0.25-\mu \mathrm{m}$ 4-metal layer CMOS technology using only core transistors. It operates with a supply voltage of $2.5 \mathrm{~V}$ and at a clock frequency of $320 \mathrm{MHz}$. The input signals are differential with a full-scale range of $\pm 500 \mathrm{mV}$ peak ( $-9 \mathrm{dBVrms})$. The functional blocks of the modulator are described next.
TABLE I

Nominal TRANSCONDUCTANCE AND CAPACITOR SizES

\begin{tabular}{|c|c|}
\hline Component & Value \\
\hline \hline$G M_{1}$ & $1000 \mu \mathrm{A} / \mathrm{V}$ \\
\hline$G M_{2}$ & $200 \mu \mathrm{A} / \mathrm{V}$ \\
\hline$G M_{3}$ & $15 \mu \mathrm{A} / \mathrm{V}$ \\
\hline$G M_{4}$ & $325 \mu \mathrm{A} / \mathrm{V}$ \\
\hline$G M_{5}$ & $24 \mu \mathrm{A} / \mathrm{V}$ \\
\hline$C_{1}$ & $5.165 p F$ \\
\hline$C_{2}$ & $0.376 p F$ \\
\hline
\end{tabular}

\section{A. Gm-C Integrators}

The integrators were built using transconductors and capacitors $(G m-C)$. This approach had the advantage of a simpler circuit structure and lower power consumption than other approaches, such as those based on operational amplifiers. In this case, the specifications for resolution and linearity are not overly demanding and allow the use of the $\mathrm{Gm}-\mathrm{C}$ approach.

The nominal transconductor and capacitor values are listed in Table I. The size of $G M_{1}, C_{1}, G M_{2}$ and $C_{2}$ were determined taking into account the contribution of thermal noise to the total ADC noise, and by making this noise nondominant (about $-6 \mathrm{~dB}$ below quantization noise) to avoid the degradation of the effective resolution of the converter. If the thermal noise is made much lower than the quantization noise, there will be a noticeable increase in power consumption. Therefore, there is a trade-off between resolution and power consumption. In this case, the resolution penalty due to thermal noise is about $1 \mathrm{~dB}$.

$G M_{3}$ was obtained from the frequency of the optimized zero of the NTF, $\omega_{o z}[16]$

$$
G M_{3}=\frac{\omega_{o z}^{2}}{G M_{2} C_{1} C_{2}}
$$

and $G M_{4}$ and $G M_{5}$ were obtained from the frequency shift of the complex modulator $\left(\omega_{\mathrm{sh}}=2 \pi \cdot 10 \mathrm{MHz}\right)$

$$
G M_{4}=\omega_{\mathrm{sh}} C_{1} \quad G M_{5}=\omega_{\mathrm{sh}} C_{2} .
$$

The transconductor schematics are shown in Fig. 6. Note that $G M_{3}$ has a different, simpler, structure than the other circuit due to its small transconductance [Fig. 6(b)]. The structure of Fig. 6(a) was described in [20]. It is a low-noise, highly linear transconductor whose normalized transconductance is shown in Fig. 7. In the -0.5 to +0.5 voltage range, the ripples of the transconductance are only about $1 \%$, providing a low distortion for the modulator. This is particularly significant for the input transconductors, because their distortion is unshaped by the loop filter. Transconductor $G M_{3}$ is implemented using MOSFETs operating in triode region. This transconductor also exhibits good linearity, although it is not highly power-efficient (lower transconductance than the transconductors of Fig. 6(a) for the same current consumption). However, the use of these transconductors does not have a noticeable impact on the total power consumption due to their small value.

Capacitors were implemented as inversion MOS capacitors. These are, in fact, N-channel MOSFET transistors with grounded source and drain terminals, and therefore neither special cells nor special technology options are required. In 
(a)

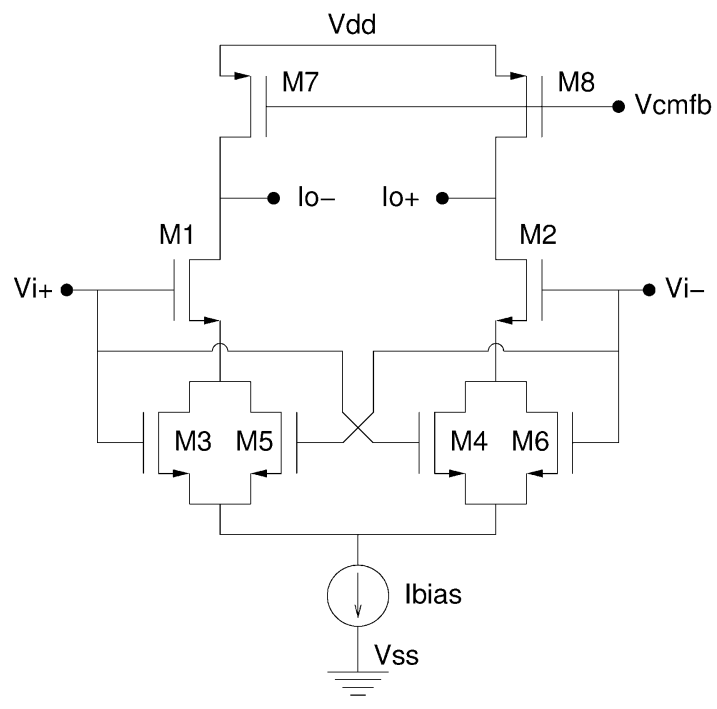

(b)

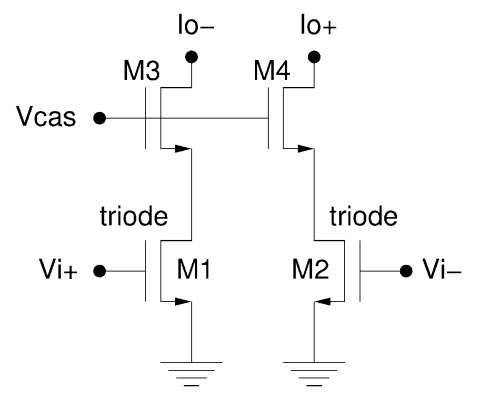

Fig. 6. Schematic of transconductors: (a) $G M_{1}, G M_{2}, G M_{4}$ and $G M_{5}$. (b) $G M_{3}$.

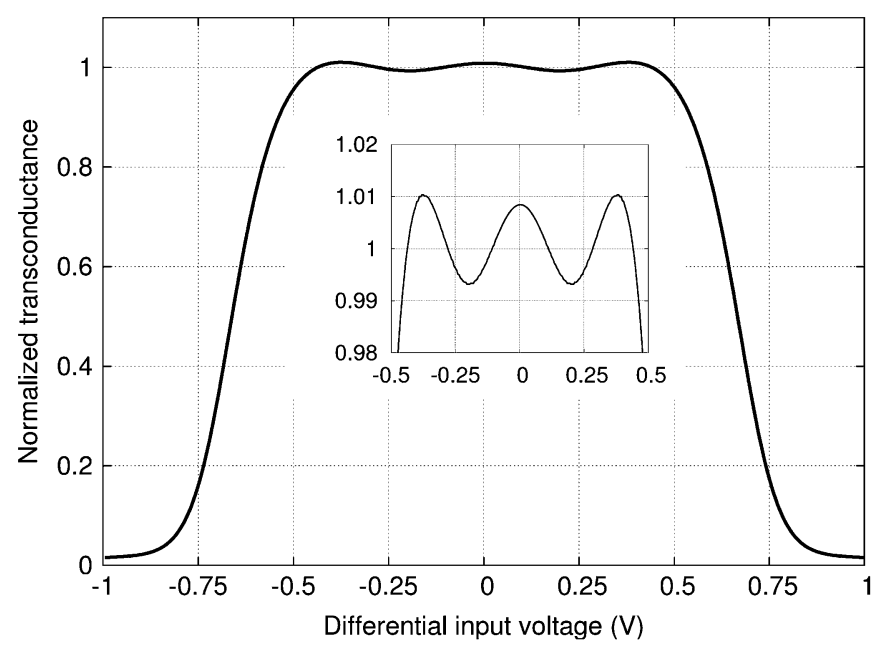

Fig. 7. Normalized transconductance for transconductors $G M_{1}, G M_{2}, G M_{4}$, and $G M_{5}$. A zoom of the upper part of the curve is shown in the inset.

addition, the high capacitance density of MOS capacitors reduces the chip area. The corresponding capacitance-voltage curve is included in Fig. 8(a) along with the output voltage ranges of the two integrators. As can be seen, the variation of the capacitance for the intended ranges is quite small, about $0.1 \%$. This distortion introduced by the nonlinear capacitance is masked by the distortion of the transconductors. In Fig. 8(b), the equivalent circuit of the capacitor is shown. The series resistance of the capacitor, $R_{S}$, depends on the common-mode voltage at the output of the integrator. Its value is

$$
R_{S} \simeq \frac{R_{\mathrm{ON}}}{4}=\frac{1}{4 K_{p} \frac{W}{L}\left(V_{\mathrm{CM}}-V_{\mathrm{TH}}\right)}
$$

where $V_{\mathrm{CM}}$ is the common-mode voltage at the integrator output, $V_{\mathrm{TH}}$ is the threshold voltage for $\mathrm{N}$-channel devices, and $W$ and $L$ are the width and length of the capacitor's gate. This (a)

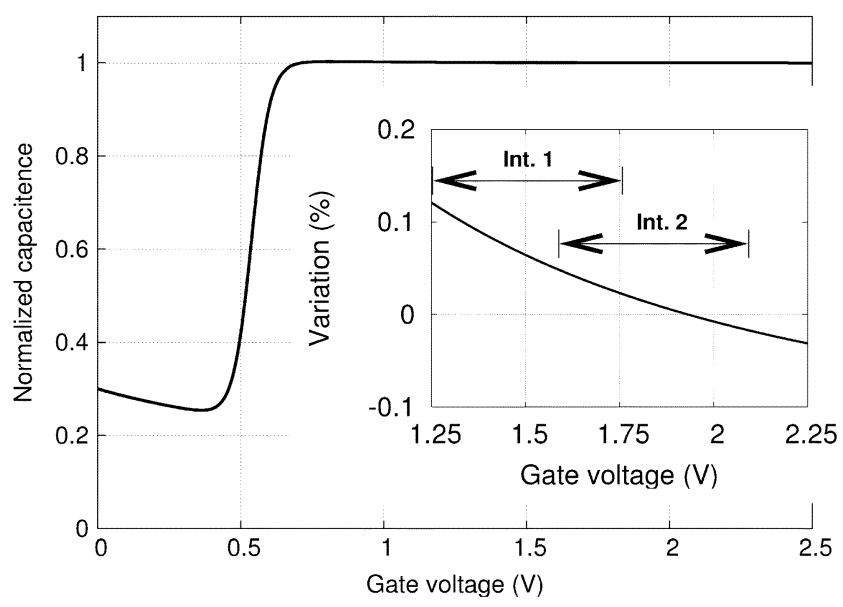

(b)
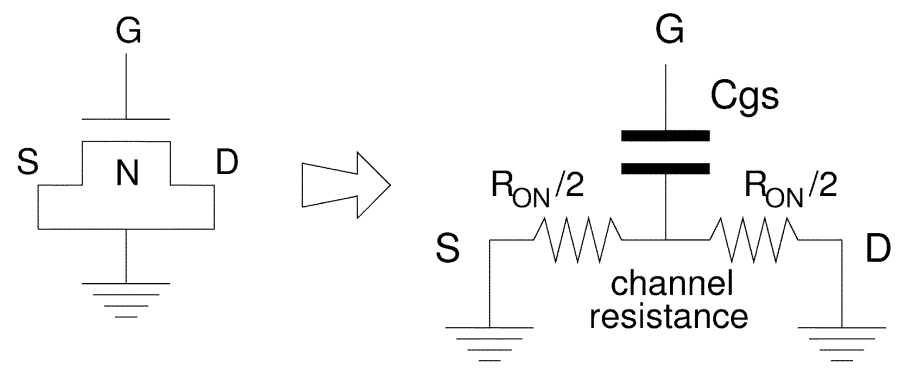

Fig. 8. MOS capacitors. (a) Capacitance versus voltage. The normalized capacitance variation along with the output voltage range of the two integrators is shown in the inset. (b) Equivalent model of the MOS capacitor.

resistance introduces a zero in the transfer function of integrators and it can change the NTF of the modulator significantly. In order to reduce this effect, the capacitors have to be split into small sections connected in parallel. In this way, the total series resistance is divided by the number of sections (if the capacitor aspect-ratio is kept constant). 

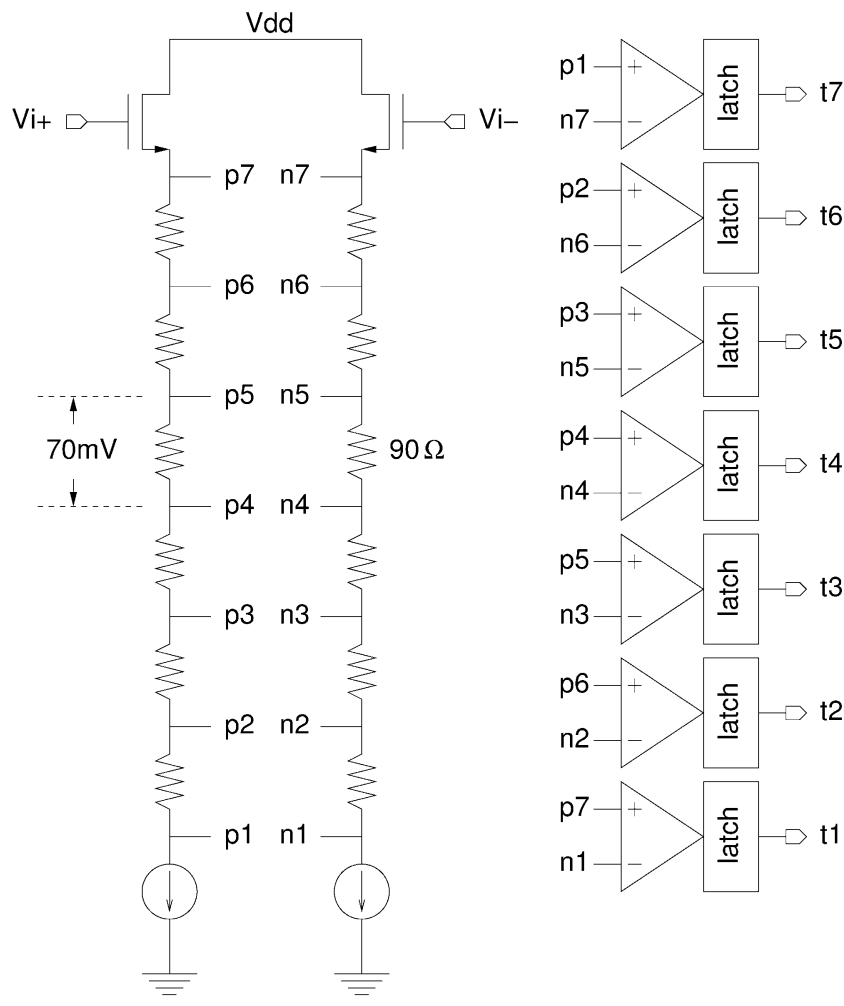

Fig. 9. Schematic of the differential-input 3-bit flash ADC.

\section{B. Flash $A D C$}

The flash ADC schematic is shown in Fig. 9. It consists of a differential input buffer, a voltage shifter based on two resistor strings, and seven regenerative comparators and latches. The resistor strings are biased by a constant current providing a $70-\mathrm{mV}$ voltage drop between consecutive taps. The value of resistors, $90 \Omega$, was chosen to give fast settling during the ADC's track phase.

The comparator schematic is shown in Fig. 10(a). It consists of a pair of input switches, a full-swing latch and two $\mathrm{C}^{2} \mathrm{MOS}$ dynamic latches. The corresponding timing is shown in Fig. 10(b). The comparator can operate in two different phases: when CLK is high, the input switches are on and internal nodes track the input voltage. Then, when CLK changes to low, the input switches turn off, sampling the input voltage, and the latch is connected to power busses. A strong positive feedback leads it to regeneration. Thus, the initial voltage difference increases exponentially until it reaches the power supply values. During the next track phase, the previous output data is held in the output latch. This latch uses a delayed clock signal to avoid disturbing regeneration at its beginning and thus reduces the effect of kick-back noise. The time-constant of the comparator is about $50 \mathrm{ps}$, and the corresponding metastability probability is one cycle every $2 \cdot 10^{10}$ cycles. The estimated offset is $8 \mathrm{mV}$.

\section{Current-Mode DACS}

Current-output DACs (Fig. 11) were built using eight identical current cells controlled by a thermometer-code input. Each cell is a cascode current source followed by three current-steering switches. Switches controlled by esn and esp (a)

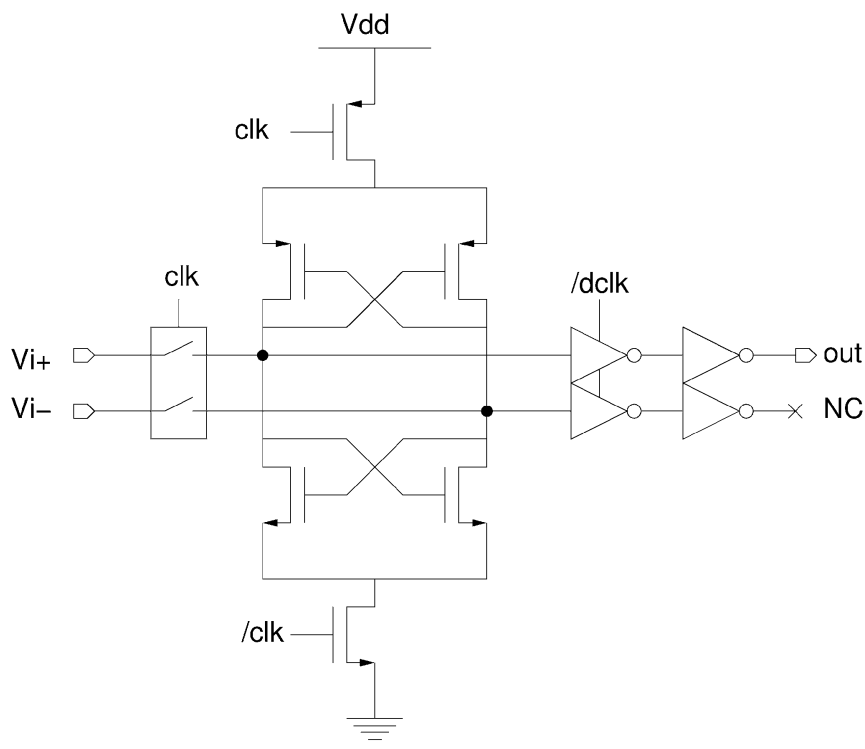

(b)

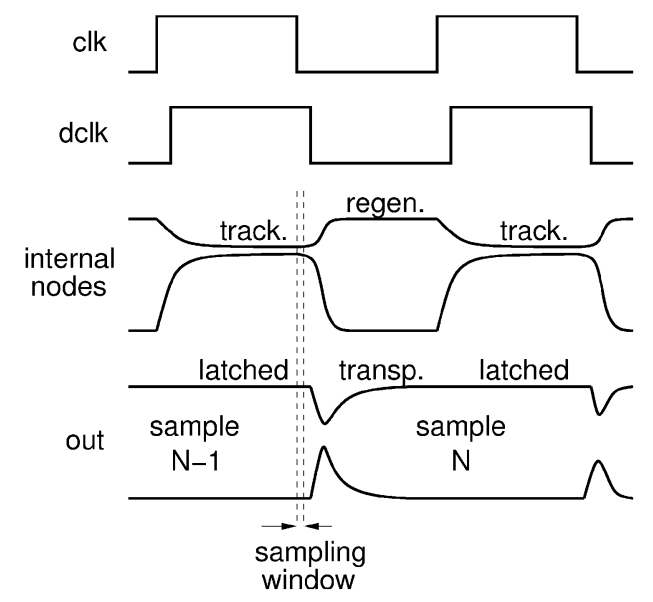

Fig. 10. (a) Schematic of comparators and latches. (b) Timing diagram.

steer the current toward the positive or negative output bus depending on the input data (these signals are generated by the digital logic discussed in the following subsection). These switches can only be on during half of the clock period (HRZ DAC) as can be seen in Fig. 11. When esp and esn are both off, the third switch, whose gate is connected to a constant reference voltage, $V_{\text {ref }}$, remains on, avoiding transients on the current source devices that are always working in saturation. This results in some power consumption penalty. However, this extra power consumption was estimated at only about $10 \%$ of the total modulator power consumption. In this off state, four cells are connected to the positive output node and four cells to the negative output one, resulting in a zero differential output current.

Since the DACs are built using only N-channel devices, they can only sink current and this has to be compensated by a proper constant current biasing implemented with P-channel devices (not shown).

The mismatch between current cells was minimized using a common-centroid layout for DACs. Also, $I$ and $Q$ channel DAC cells are interlaced to improve image rejection. The effect 


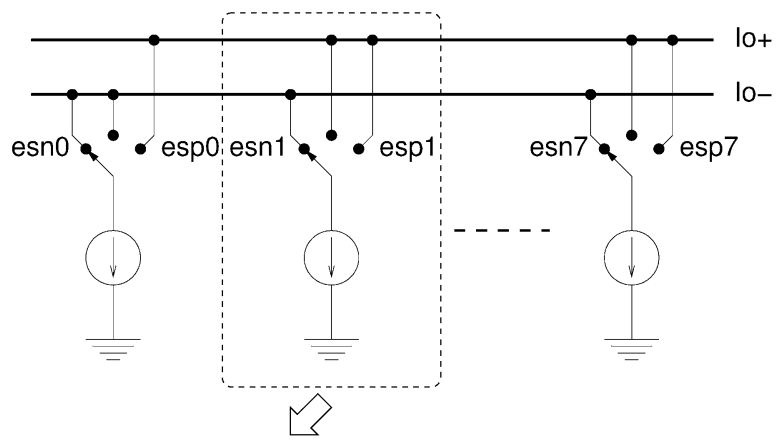

Current cell

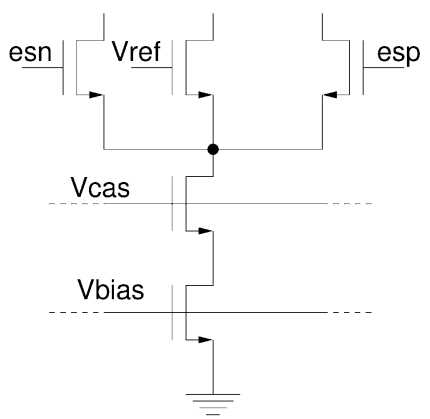

Signal timing

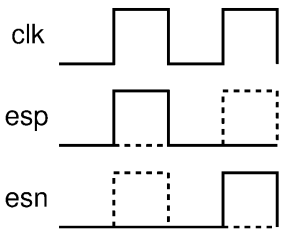

Fig. 11. Schematic of current-output DACs and related signal timing.

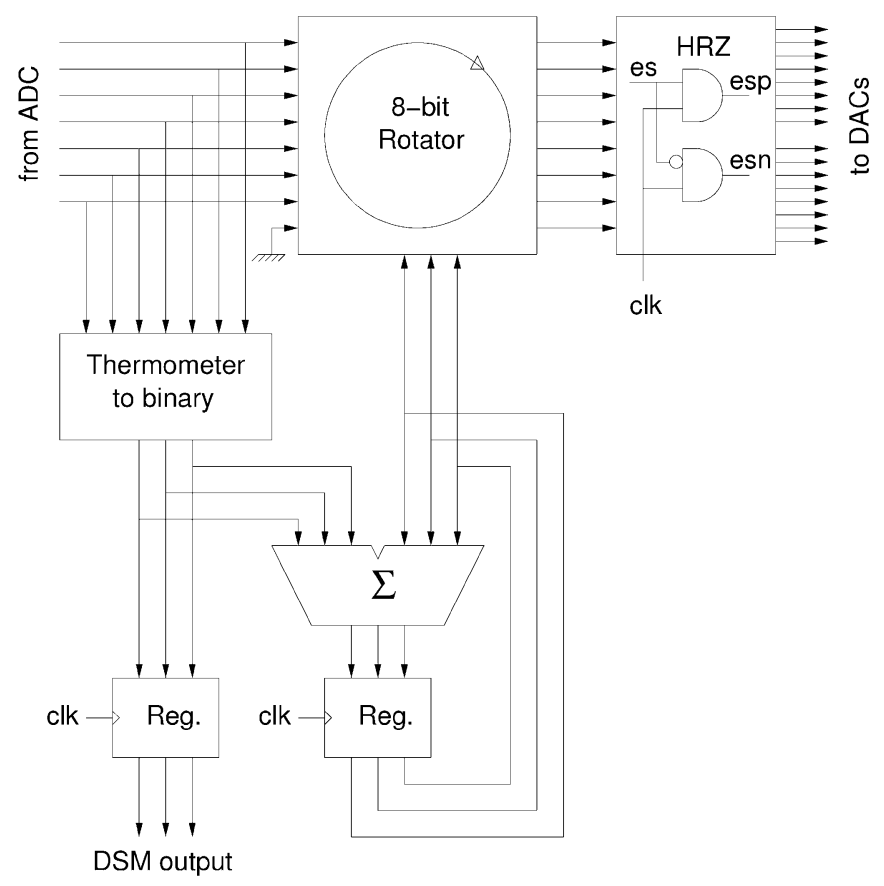

Fig. 12. Block diagram of the digital logic of a real modulator.

of these mismatches is further reduced by the DWA algorithm whose implementation is presented in the next subsection.

\section{Digital Logic}

Certain parts of the modulator are implemented in the digital domain. The digital logic diagram is shown in Fig. 12. It includes a thermometer-to-binary-code converter, an 8-bit combinatorial rotator and the associated DWA logic, and the HRZ logic. The DWA algorithm is based on the rotation of the thermometer-code output with a rotation index that is incremented

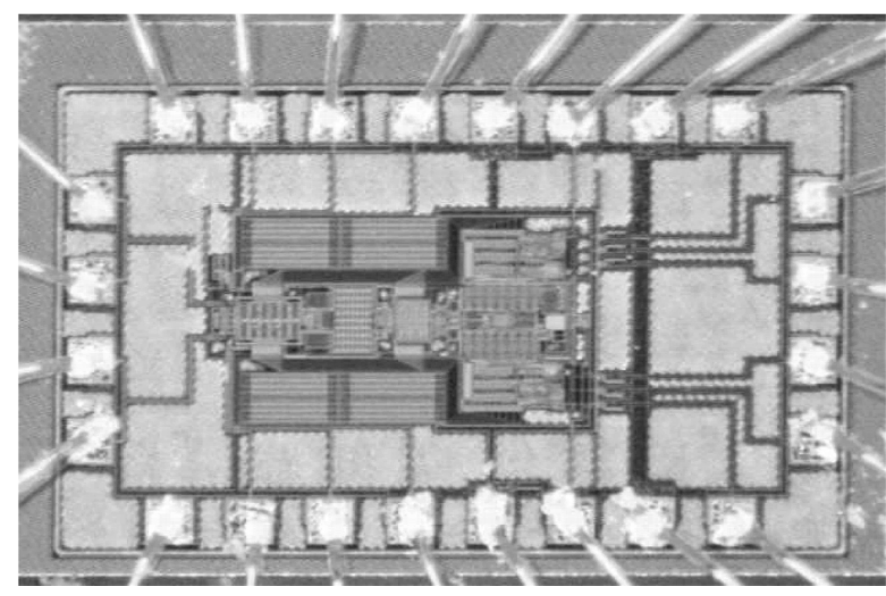

Fig. 13. Photograph of the test chip bonded to a PCB.

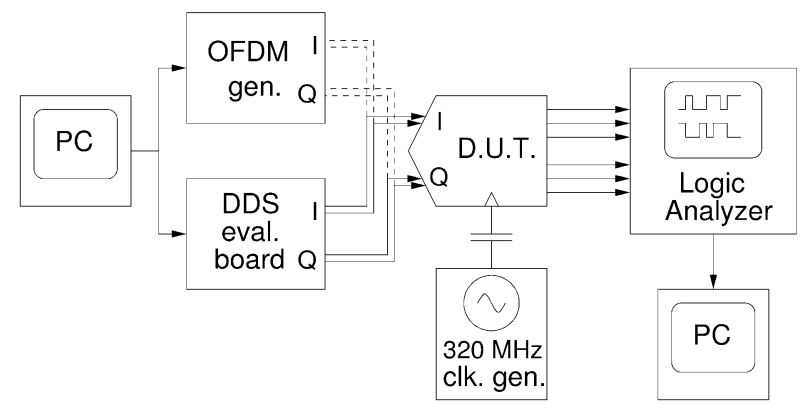

Fig. 14. Experimental setup for measurements.

by the value of the modulator output on each sample. In this way, the nonlinearity of DACs is translated into a first-order shaped noise floor owing to the element scrambling. The HRZ logic generates the signals to drive the DAC inputs.

\section{EXPERIMENTAL RESULTS}

The converter was fabricated and a test chip was bonded to a PCB using a room-temperature conductive glue; the chip photograph is shown in Fig. 13. The chip area is $1480 \times 880 \mu \mathrm{m}^{2}$ including pads. Without pads, the active area is reduced by $2 / 3$. The chip was powered by three separate power supplies, all operating at $2.5 \mathrm{~V}$. A power supply was provided for analog circuitry, another power supply was used for digital circuitry, and the third power supply was used only for output pin drivers. The power consumption, not including pin drivers, was $30.4 \mathrm{~mW}$ for the modulator operating in the ZIF mode and $32 \mathrm{~mW}$ for LIF mode. This small difference is due to the coupling transconductors (Fig. 4) that are disabled in the ZIF mode. The power consumption of the digital blocks was $12 \mathrm{~mW}$ in both modes, and the power consumption of the pin drivers was about $10 \mathrm{~mW}$. This latter figure is highly dependent on the load capacitance.

The experimental setup for measurements is shown in Fig. 14. The input is a complex differential signal obtained either from a direct digital synthesizer (DDS) or from a programmable vector signal generator, capable of generating OFDM signals. The clock signal is obtained from a $320-\mathrm{MHz}$ sine-wave RF generator. The output bits are acquired by a high-speed logic analyzer and then transferred to a computer for further processing. 


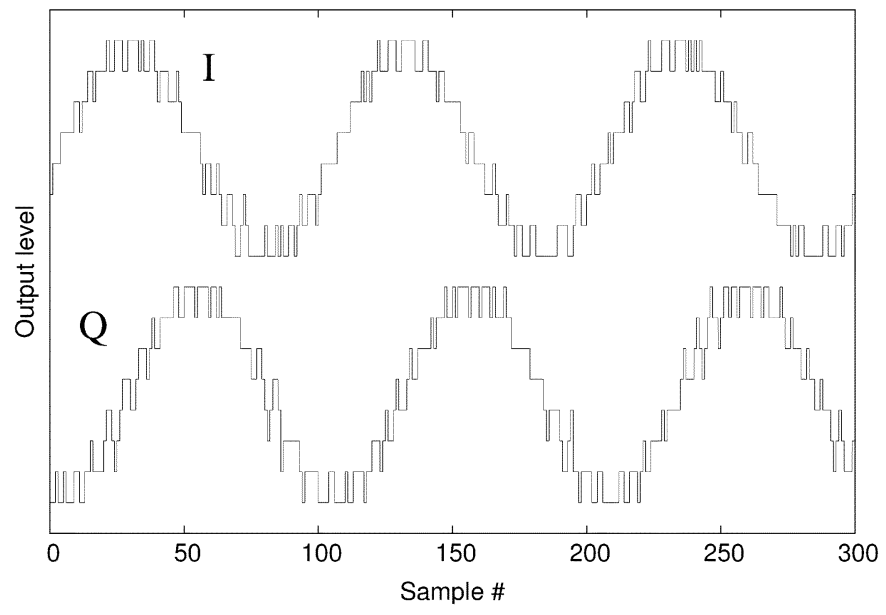

Fig. 15. Time-domain output for a complex, $+3.125-\mathrm{MHz}$ full-scale sine-wave input. (The waveforms are shifted on the Y-axis for the sake of clarity).

\section{A. Sine-Wave Based Measurements}

A set of measurements were performed using complex sinewave input signals. These signals were obtained from an Analog Devices AD9854 DDS evaluation board. To allow for image-rejection measurements, the input signal to the modulator had to be a complex sine-wave with accurate quadrature. The DDS output already provides high phase accuracy, but some calibration was needed in order to cancel the gain imbalance of $I$ and $Q$ outputs. After calibration, the output presents only a positive frequency, with more than $60 \mathrm{~dB}$ of image frequency attenuation, $13 \mathrm{~dB}$ higher than the measured image rejection of the test chip.

In Fig. 15, an example of the output waveforms is shown. The output sine-wave is distorted by quantization noise, and parameter extraction is difficult. Therefore, the output signal is translated into the frequency domain using FFT.

When the cross-coupling transconductors (Fig. 4) are disconnected (ZIF mode), the modulator can be viewed as two independent real, single-input modulators. The corresponding output for these modulators is shown in Fig. 16(a) for a $3.125-\mathrm{MHz}-13-\mathrm{dBV}$ single-tone input. When the two modulators are considered as a complex modulator, the corresponding output includes both positive and negative frequencies [Fig. 16(b)]. The noise floor shape follows the designed NTF where the two zeros are still visible, although they are filled with noise due to several nonideal effects including finite integrator gain, thermal noise and clock jitter. In addition to the input test tone, several other tones are present at the output. The observed DC level corresponds to about $10 \mathrm{mV}$. The image frequency (IM) at $-3.125 \mathrm{MHz}$ is $47.2 \mathrm{~dB}$ lower than the input signal. This high image rejection is achieved mainly due to the proper layout of the circuit and it is high enough to operate the receiver without any ADC calibration. Several harmonics can also be observed in the output spectrum, but their amplitudes are small. They are mainly originated by the input transconductor, according to simulations using its nonlinear transfer characteristic. The total harmonic distortion (THD) for $-13 \mathrm{dBV}$ input is $-58 \mathrm{dBc}$. The measured SNR for this input

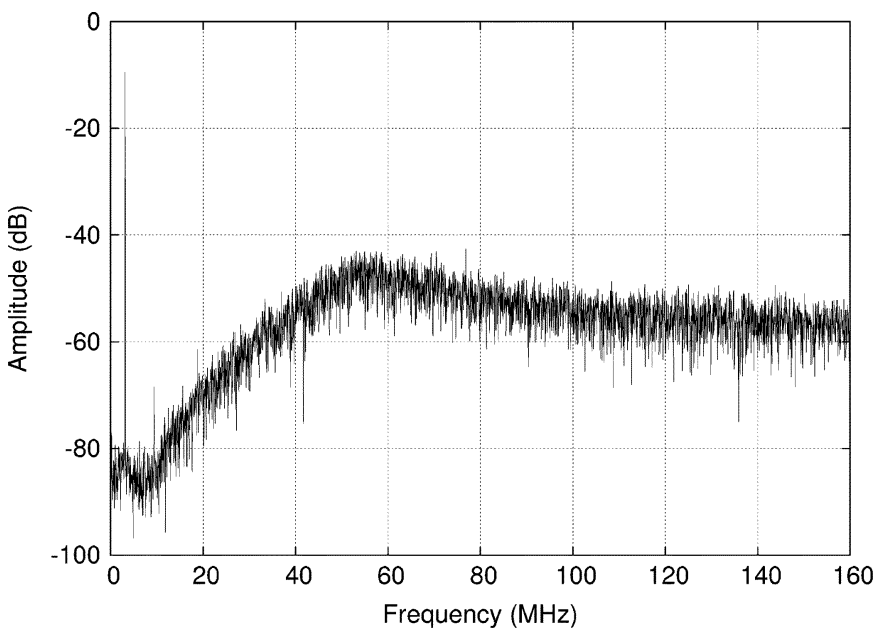

(a)

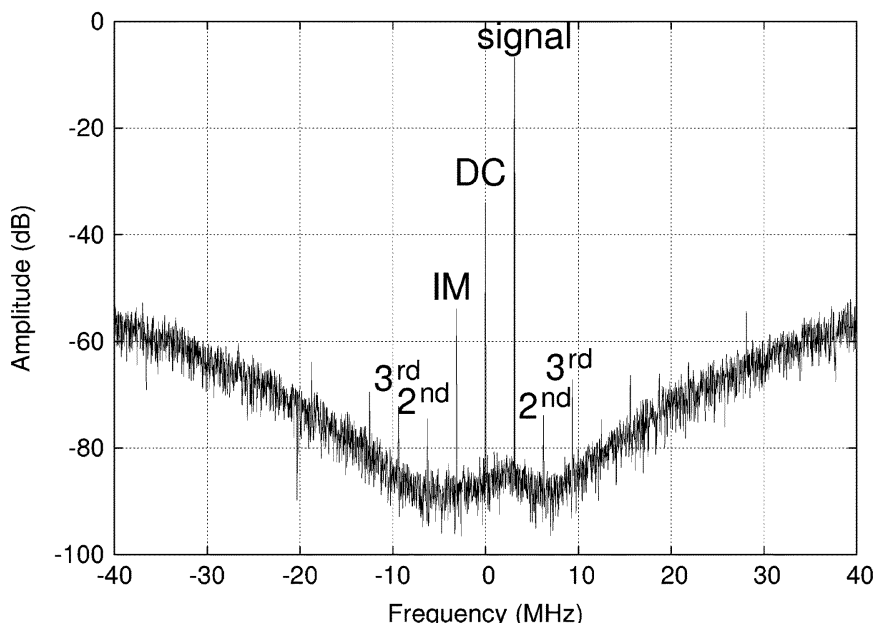

(b)

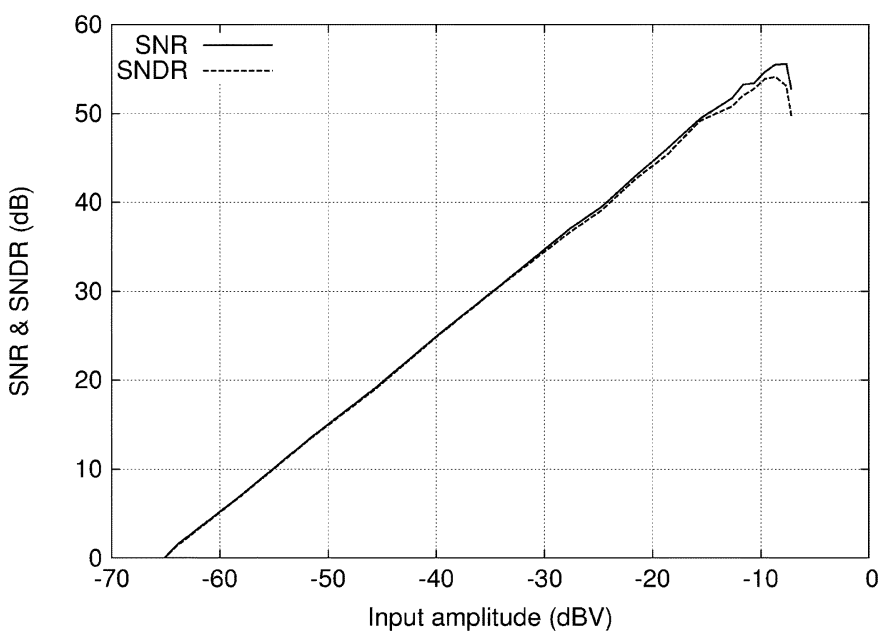

(c)

Fig. 16. Output spectra for (a) single real modulator, (b) complex ZIF-mode modulator (detail), and (c) SNR and SNDR plots as a function of the input amplitude.

amplitude is $51.8 \mathrm{~dB}$. In Fig. 16(c) the SNR and SNDR of the complex modulator in ZIF mode are plotted as a function of the input amplitude. A peak SNR and SNDR of $55.5 \mathrm{~dB}$ and $53.9 \mathrm{~dB}$, respectively, are obtained for an input amplitude of 


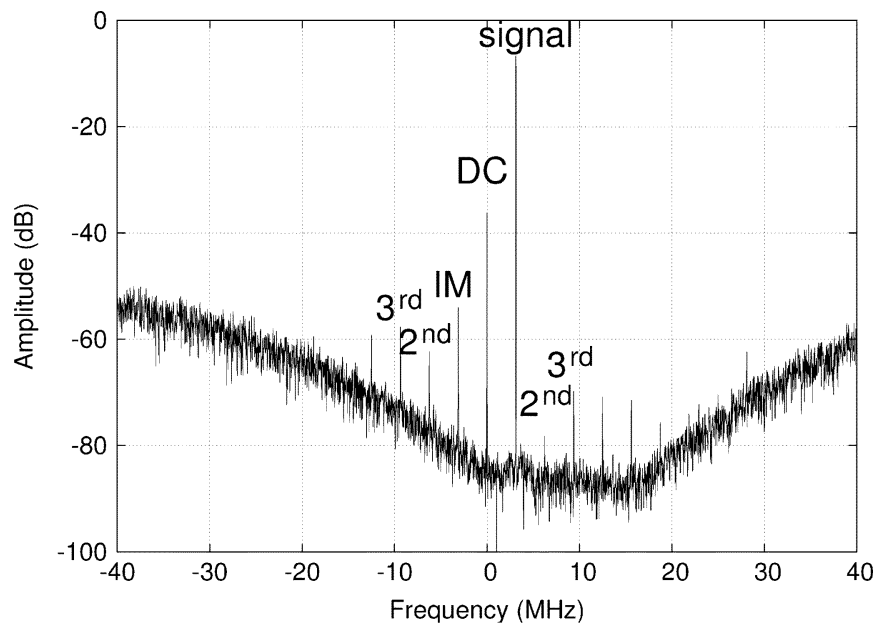

(a)

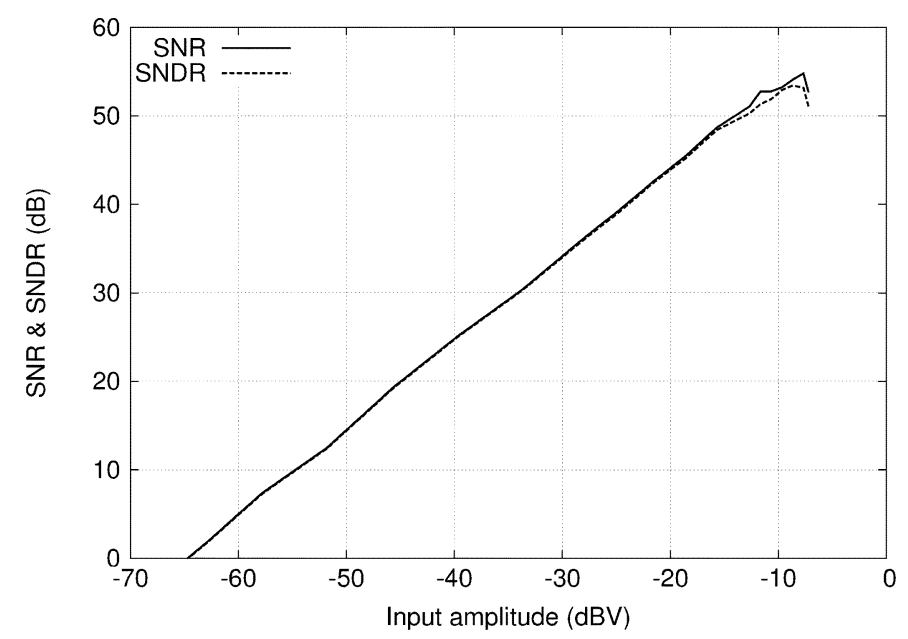

(b)

Fig. 17. (a) Output spectrum for complex, LIF mode, modulator (detail), and (b) SNR and SNDR plots as a function of the input amplitude.

$-9 \mathrm{dBV}$. The effect of distortion is small and only noticeable at high input amplitudes.

By enabling the cross-coupling transconductors, the NTF of the modulator is shifted $+10 \mathrm{MHz}$ (LIF mode). The output spectrum for an input amplitude of $-13 \mathrm{dBV}$ is shown in Fig. 17(a). In this plot, the first NTF minimum is not obvious because of the noise around the input tone, but it is visible for lower input amplitudes. Also, the frequency shift is not exactly $10 \mathrm{MHz}$ due to the inaccurate coupling between the real and imaginary channels of the modulator, but the impact on the ADC performance is not noticeable. The DC component corresponds to $8 \mathrm{mV}$. The image rejection is the same as for the ZIF mode's $47.2 \mathrm{~dB}$, showing better channel matching than that assumed in simulations. For this amplitude, the THD is $-58.3 \mathrm{dBc}$ and the SNR is $51.1 \mathrm{~dB}$. In Fig. 17(b), the SNR and SNDR of the modulator operating in LIF mode is plotted as a function of the input amplitude. The peak values for SNR and SNDR are $54.5 \mathrm{~dB}$ and $53.5 \mathrm{~dB}$, respectively, for $-9-\mathrm{dBV}$ input amplitude.

The effect of distortion can also be checked using a two-tone test. In Fig. 18 the output spectrum for a two tone input is shown. The input amplitude of each tone was half of that corresponding to the peak SNR value from Fig. 17(b) (i.e., -15 dBV). The

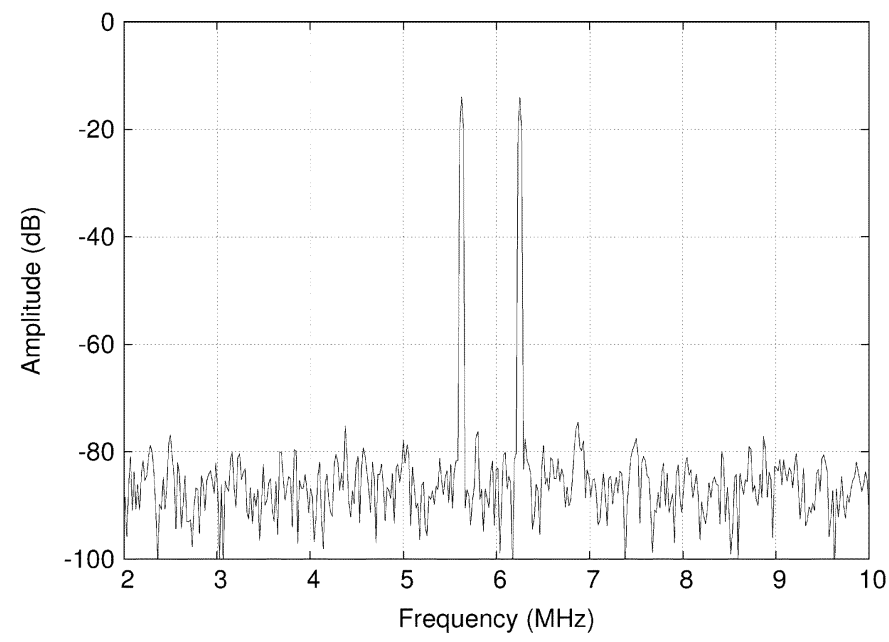

Fig. 18. Intermodulation test. Output spectrum for a two-tone input.

intermodulation products are barely visible, giving a spuriousfree dynamic range of more than $60 \mathrm{~dB}$.

The measured SNR and SNDR are less than $1 \mathrm{~dB}$ below the results obtained from a transistor-level simulation using the extracted circuit from the chip layout. These simulations did not include thermal noise. Therefore, thermal noise level is not responsible for performance degradation. The observed SNR decrease with respect to that of an ideal modulator can be attributed to the combined effect of several nonidealities such as finite DC gain in integrators, excess loop delay and clock jitter.

\section{B. Clock Jitter Measurements}

The observed SNR depends on the clock input amplitude. The clock signal was a sine-wave obtained from an RF generator. This sine-wave is amplified by the input CMOS inverter resulting in an internal clock that is an almost trapezoidal waveform with sharp edges. The input clock has a finite slew-rate, which is proportional to its amplitude. If noise is present at the clock input, it is translated into internal clock jitter that can degrade the SNR.

The jitter generated by noise at the clock input is

$$
\sigma_{\mathrm{jitter}}=\frac{T \sigma_{v}}{2 \pi A}
$$

where $\sigma_{v}$ is the equivalent input voltage noise (rms) and $A$ is the clock signal amplitude. By combining this (8) with (4), and including an additional, uncorrelated, constant source of noise, $N$, which accounts for quantization noise and all other sources of noise, the following relationship is obtained:

$$
\mathrm{SNR}(\mathrm{dB})=-10 \cdot \log _{10}\left[2\left(\frac{\sigma_{v}^{2}}{4 \pi^{2} A^{2}}+N^{2}\right)\right] .
$$

In Fig. 19, the measured SNR for a single tone input of $-13 \mathrm{dBV}$ is plotted as a function of the clock amplitude together with a curve fit to (9). The agreement with experimental data is excellent. This fit gives a value for $\sigma_{v}$ of about $6 \mathrm{mV} \mathrm{rms}$, which suggests that the equivalent input noise of the clock is dominated by power supply noise rather than thermal noise. In order to reduce the effect of clock jitter the final clock signal used for 


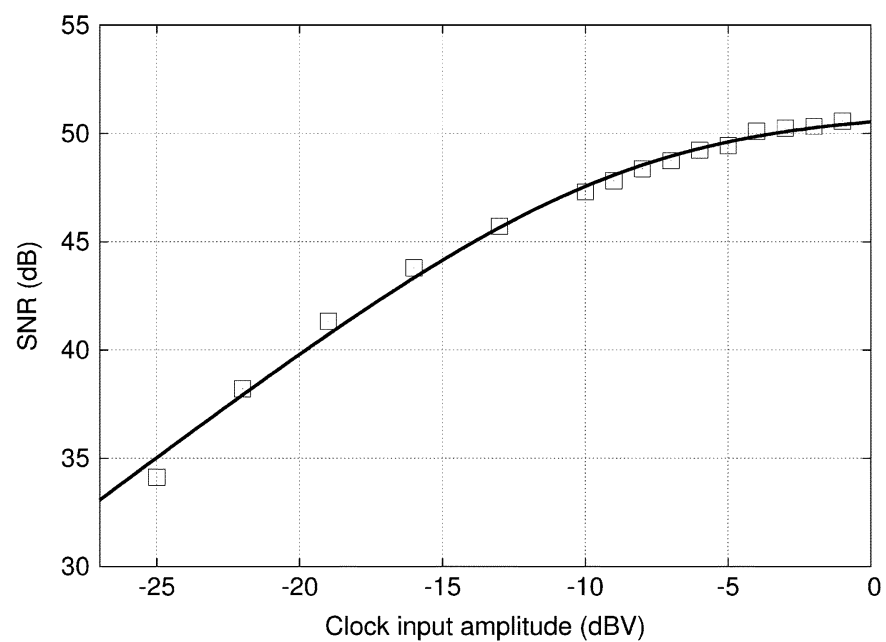

Fig. 19. SNR as a function of the clock amplitude (squares). The clock signal is a sine-wave. Test tone amplitude was $-13 \mathrm{dBV}$. A curve fit to Eq. (9) is included as a solid line.

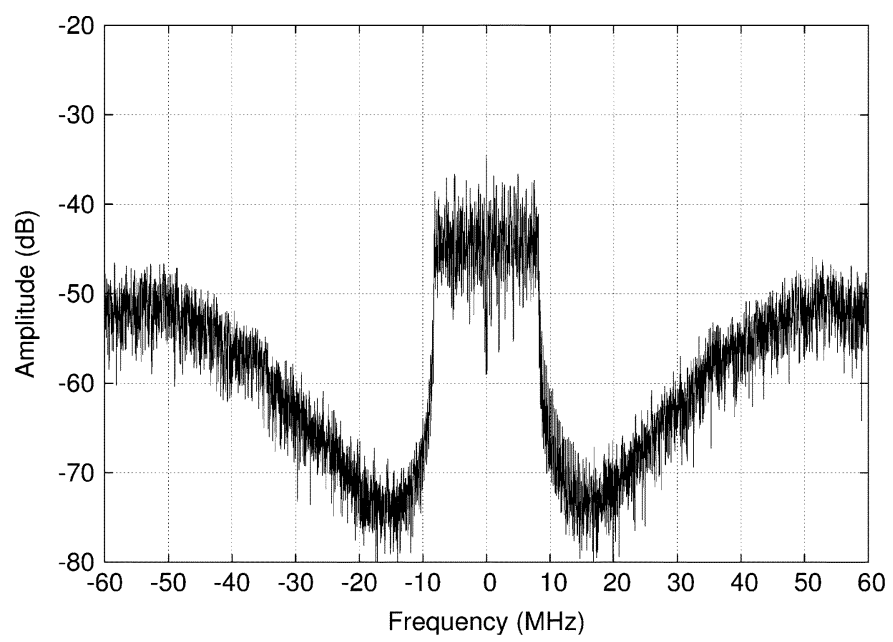

(a)

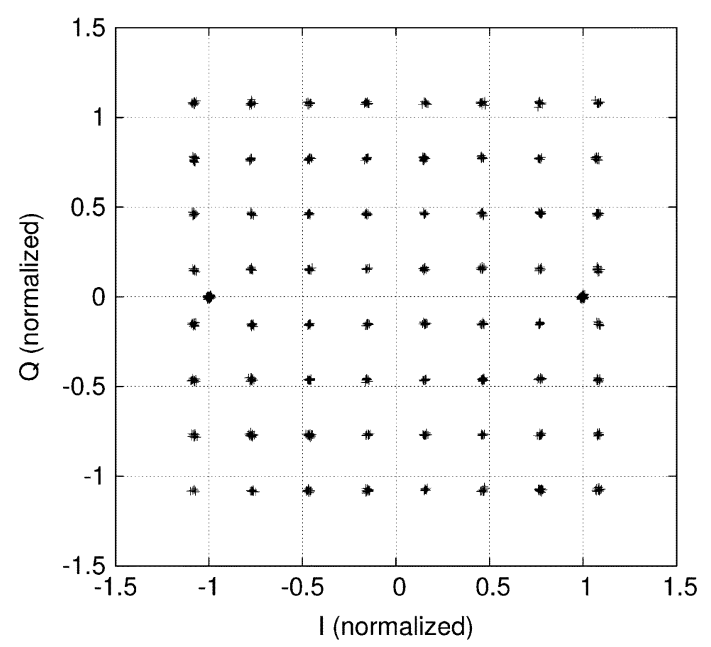

(b)

Fig. 20. (a) Output OFDM spectrum for ZIF mode. (b) Its corresponding constellation.

all measurements was an amplified and clipped sine-wave with sharp edges, giving an estimate for jitter of $\sigma_{\text {jitter }}=0.75 \mathrm{ps}$ $\left(\sigma_{\text {jitter }} / T=0.024 \% \mathrm{rms}\right)$.

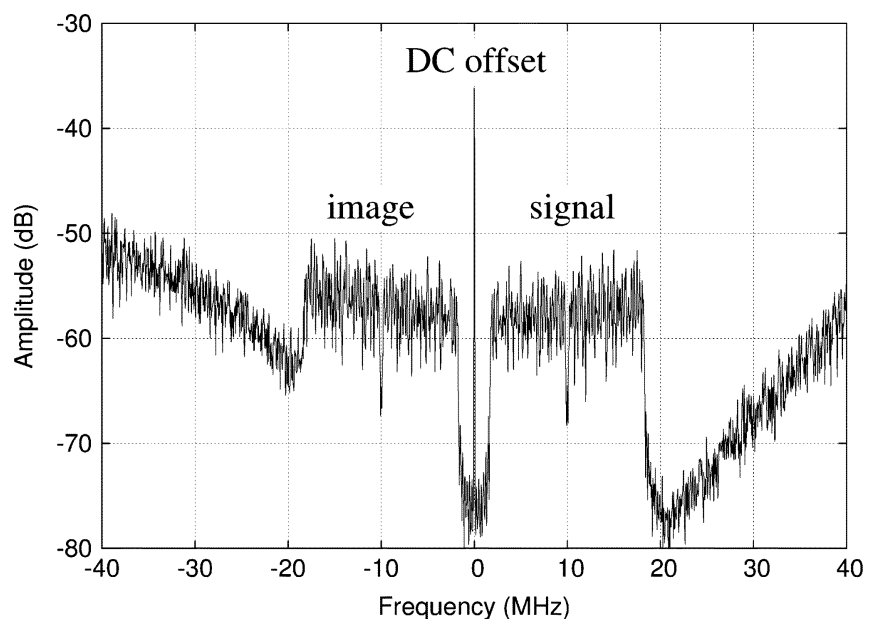

(a)

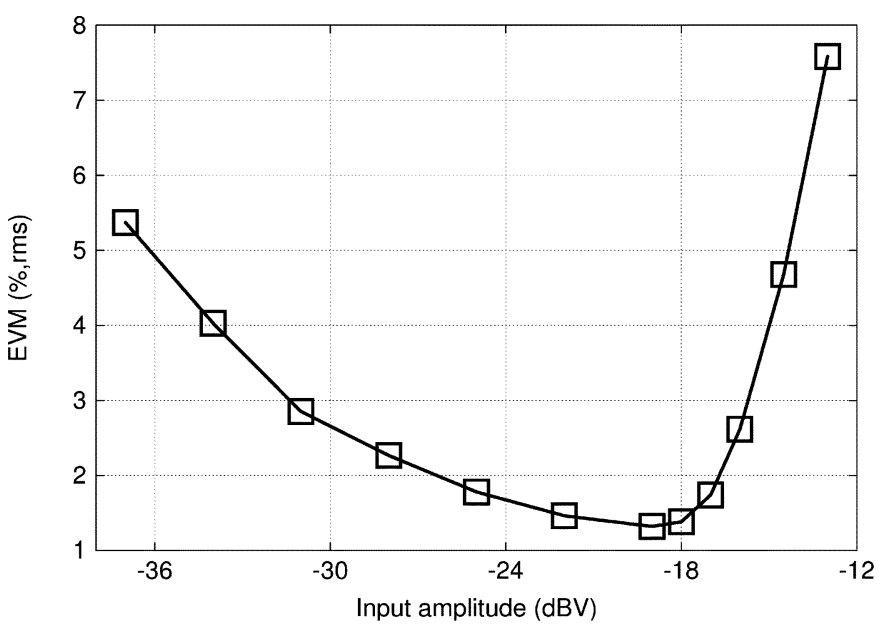

(b)

Fig. 21. (a) Output spectrum for LIF mode. The input signal is a real OFDM signal modulated at $10 \mathrm{MHz}$ and with $-37 \mathrm{dBV}$ amplitude. (b) EVM as a function of the input amplitude for LIF mode.

\section{OFDM Based Measurements}

A set of measurements were carried out using modulated OFDM signals in order to test the performance of the modulator for the intended application. In Fig. 20(a), the spectrum of a converted baseband OFDM signal is shown with the modulator working in ZIF mode. After demodulating the converted signal, the corresponding constellation of Fig. 20(b) is obtained. The error vector magnitude (EVM) for all subcarriers is about $1 \%$ rms, well below the maximum $5.6 \%$ required for an error-free reception [21].

Similar measurements were done for the LIF mode. In this case, the OFDM signal was modulated with a center frequency of $+10 \mathrm{MHz}$. The signal generator available provided a single RF output, which was connected to the $I$-channel input of the $\mathrm{ADC}$, while the $Q$-channel input was grounded. This experimental setup reduced the peak SNR by $3 \mathrm{~dB}$ with respect to an ADC with a complex input. Moreover, the negative frequencies are also present at the input reducing further the available dynamic range for the desired signal band. The measured spectrum is shown in Fig. 21(a). The image band has to be rejected by digital filtering using a complex decimator/filter. Alternatively, a $-10 \mathrm{MHz}$ frequency shift (mixing) may be performed prior 


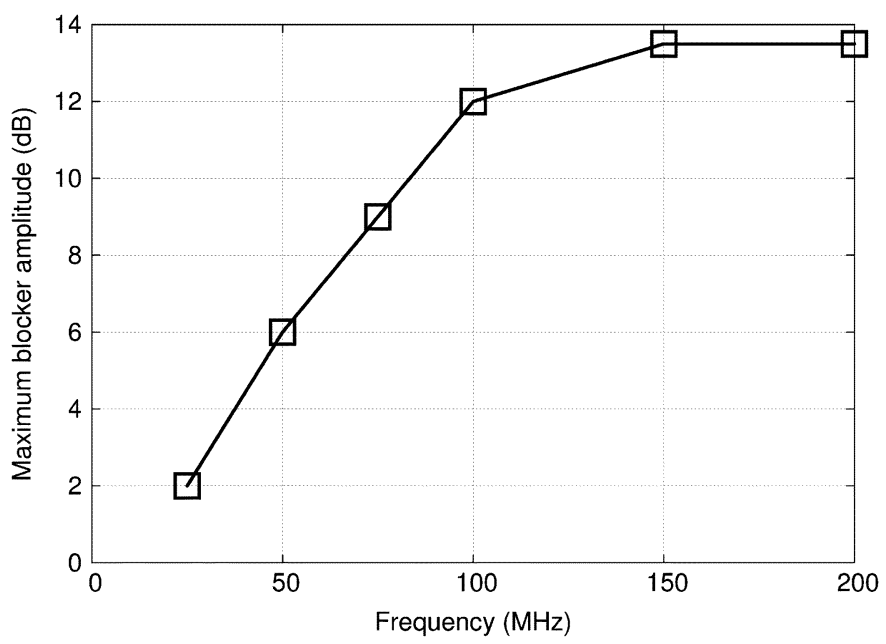

Fig. 22. Maximum allowed out-of-band blocker amplitude, relative to signal, for different frequencies and for LIF mode. Input amplitude: $-19 \mathrm{dBV}$.

to filtering, and then, a conventional decimator/filter would suffice. By computing the corresponding constellation for different input amplitudes, and extracting the EVM, the plot of Fig. 21(b) was obtained. The minimum EVM value is about $1.3 \%$ for an input amplitude of $-19 \mathrm{dBV}$.

Finally, an out-of-band, sine-wave interferer (blocker) was added to the $10 \mathrm{MHz}$-modulated OFDM signal. The amplitude of this blocker was adjusted to obtain an EVM of 3\%. The maximum blocker amplitude relative to the signal amplitude is plotted for several frequencies in Fig. 22. For frequencies far away from the signal band, maximum amplitudes higher than $13 \mathrm{~dB}$ are allowed. This graph, together with the interference mask specifications [21], can be used to determine the required filter that must precede the $\Delta \Sigma$ modulator.

CT modulators also exhibit implicit anti-alias filtering [6]. The measured alias frequency rejection was about $49 \mathrm{~dB}$ for ZIF mode and $47 \mathrm{~dB}$ for LIF mode. These values were found to be almost constant throughout the whole alias band (310 to $330 \mathrm{MHz}$ for ZIF mode, and 300 to $340 \mathrm{MHz}$ for LIF mode).

\section{CONCLUSION}

A complex multi-bit continuous-time delta-sigma modulator intended for IEEE $802.11 \mathrm{a} / \mathrm{b} / \mathrm{g}$ wireless LAN receivers was designed, fabricated and evaluated. This modulator runs at $320 \mathrm{MHz}$ with an oversampling ratio of 16 for an input signal bandwidth of $20 \mathrm{MHz}$. It was designed in $0.25-\mu \mathrm{m}$ standard CMOS technology using a $2.5-\mathrm{V}$ power supply. It achieves approximately $55 \mathrm{~dB}$ of peak signal to noise ratio for complex signals. The modulator can operate on two modes: ZIF and LIF. In the ZIF mode it acts as two independent real (single input) modulators and it exhibits a NTF that is symmetrical around DC. In the LIF mode, the two real modulators are cross-coupled and the resulting NTF is shifted $+10 \mathrm{MHz}$, making it adequate for the optimal digitization of signals from low-IF radio receivers. The performance of the complex modulator was tested experimentally using both sine-wave signals and modulated OFDM signals, proving that it meets the requirements for the intended application. The achievable SNR, together with the
TABLE II

MOdULATOR PERFORMANCE

\begin{tabular}{|c|c|c|}
\hline Technology & \multicolumn{2}{|c|}{$0.25 \mu \mathrm{m} 4 \mathrm{M} 1 \mathrm{P}$ CMOS } \\
\hline Area w. pads & \multicolumn{2}{|c|}{$1.30 \mathrm{~mm}^{2}$} \\
Area w.o. pads & \multicolumn{2}{|c|}{$0.44 \mathrm{~mm}^{2}$} \\
\hline Supply voltage & \multicolumn{2}{|c|}{$2.5 \mathrm{~V}$} \\
\hline Experimental results & $\begin{array}{c}\text { ZIF mode } \\
(-10 \ldots+10 \mathrm{MHz})\end{array}$ & $\begin{array}{c}\text { LIF mode } \\
(0 \ldots+20 \mathrm{MHz})\end{array}$ \\
\hline Power consumption & & \\
Analog & $18.4 \mathrm{~mW}$ & $20.0 \mathrm{~mW}$ \\
Digital & $12.0 \mathrm{~mW}$ & $12.0 \mathrm{~mW}$ \\
Total & $30.4 \mathrm{~mW}$ & $32.0 \mathrm{~mW}$ \\
\hline peak SNR & $55.5 \mathrm{~dB}$ & $54.5 \mathrm{~dB}$ \\
\hline peak SNDR & $53.9 \mathrm{~dB}$ & $53.5 \mathrm{~dB}$ \\
\hline ENOB & $8.9 \mathrm{bits}$ & $8.7 \mathrm{bits}$ \\
\hline Image rejection & $47.2 \mathrm{dBc}$ & $47.2 \mathrm{dBc}$ \\
\hline DC offset & $10 \mathrm{mV}$ & $8 \mathrm{mV}$ \\
\hline EVM (min) & $<1.0 \%$ & $1.3 \%$ \\
\hline Blocker rejection & & \\
@ 25 MHz & - & $2 \mathrm{~dB}$ \\
@ 50 MHz & - & $6 \mathrm{~dB}$ \\
@ 100 MHz & - & $12 \mathrm{~dB}$ \\
\hline Alias rejection & $49 \mathrm{~dB}$ & $47 \mathrm{~dB}$ \\
\hline
\end{tabular}

low distortion, high image frequency rejection, moderate power consumption and relaxed pre-filtering requirements makes this architecture a promising option for high-performance and low-cost, multi-mode, wireless-LAN receivers. The measured modulator performance is summarized in Table II.

\section{ACKNOWLEDGMENT}

The authors would like to acknowledge the anonymous reviewers for their useful and detailed feedback, which considerably improved the quality of this manuscript.

\section{REFERENCES}

[1] S. R. Norsworthy, R. Schreier, and G. C. Temes, Eds., Delta-Sigma Data Converters: Theory, Design, and Simulation. New York: IEEE Press, 1996.

[2] R. T. Baird and T. S. Fiez, "Linearity enhancement of multibit deltasigma $\mathrm{A} / \mathrm{D}$ and $\mathrm{D} / \mathrm{A}$ converters using data weighted averaging," IEEE Trans. Circuits Syst. II, Analog Digit. Signal Process., vol. 42, no. 12, pp. 753-762, Dec. 1995

[3] R. Schreier and B. Zhang, "Noise-shaped multi-bit D/A converter employing unit elements," Electron. Lett., vol. 31, no. 20, pp. 1712-1713, Sep. 1995.

[4] R. Mittal and D. J. Allstot, "Low-power high-speed continuous-time sigma-delta modulators," Proc. IEEE ISCAS, pp. 183-186, 1995.

[5] E. J. van der Zwan and E. C. Dijkmans, "A 0.2-mW CMOS sigma-delta modulator for speech coding with $80 \mathrm{~dB}$ dynamic range," IEEE J. SolidState Circuits, vol. 31, no. 12, pp. 1873-1880, Dec. 1996.

[6] J. A. Cherry and W. M. Snelgrove, Continuous-Time Delta-Sigma Modulators for High Speed A/D Conversions. Boston, MA: Kluwer Academic, 2000.

[7] P. Vancorenland, P. Coppejans, W. de Cock, and M. Steyaert, "A quadrature direct digital downconverter," in Proc. IEEE Custom Integrated Circuits Conf., 2002, pp. 235-238.

[8] K. Philips, "A $4.4 \mathrm{~mW} 76 \mathrm{~dB}$ complex delta-sigma ADC for Bluetooth receivers," in IEEE ISSCC Dig. Tech. Papers, Feb. 2003, pp. 64-65.

[9] L. Dorrer, F. Kuttner, A. Wiesbauer, A. Di Giandomenico, and T. Hartig, "10-bit, 3-mW continuous-time sigma-delta ADC for UMTS in a $0.12-\mu$ m CMOS process," in Proc. Eur. Solid-State Circuits Conf., 2003, pp. 245-248.

[10] R. van Veldhoven, "A triple-mode continuous-time sigma-delta modulator with switched-capacitor feedback DAC for a GSM/EDGE/CDMA2000/UMTS receiver," IEEE J. Solid-State Circuits, vol. 38, no. 12, pp. 2069-2076, Dec. 2003. 
[11] M. Moyal, M. Groepl, H. Werker, G. Mitteregger, and J. Schambacher, "A 700/900 mW/channel CMOS dual analog front-end IC for VDSL with integrated 11.5/14.5 dBm line drivers," in Proc. IEEE Int. SolidState Circuits Conf., 2003, pp. 416-504.

[12] L. J. Breems, R. Rutten, and G. Wetzker, "A cascaded continuous-time sigma-delta modulator with $67-\mathrm{dB}$ dynamic range in $10-\mathrm{MHz}$ bandwidth," IEEE J. Solid-State Circuits, vol. 39, no. 12, pp. 2152-2160, Dec. 2004.

[13] S. Paton, A. Di Giandomenico, L. Hernández, A. Wiesbauer, P. Potscher, and M. Clara, "A 70-mW 300-MHz CMOS continuous-time sigma-delta ADC with 15-MHz bandwidth and 11-bits of resolution," IEEE J. SolidState Circuits, vol. 39, no. 7, pp. 1056-1062, Jul. 2004.

[14] A. Tabatabaei, K. Onodera, M. Zargari, H. Samavati, and D. K. Su, "A dual channel sigma-delta ADC with $40 \mathrm{MHz}$ aggregate signal bandwidth," in IEEE Int. Solid-State Circuits Conf. Dig. Tech. Papers, 2003, pp. $66-78$.

[15] S. A. Jantzi, K. W. Martin, and A. S. Sedra, "Quadrature bandpass deltasigma modulation for digital radio," IEEE J. Solid-State Circuits, vol. 32, no. 12, pp. 1935-1950, Dec. 1997.

[16] R. Schreier, "An empirical study of high-order single-bit delta-sigma modulators," IEEE Trans. Circuits Syst. II, Analog Digit. Signal Process., vol. 40, no. 8, pp. 461-466, Aug. 1993.

[17] H. Aboushady, "Design for reuse of current-mode continuous-time sigma-delta analog-to-digital converters," Ph.D. dissertation, Dept. Electron., Commun. Comput. Sci., Univ. Paris VI, Paris, France, Jan. 2002.

[18] L. J. Breems, E. C. Dijkmans, and J. H. Huijsing, "A quadrature datadependent DEM algorithm to improve image rejection of a complex sigma-delta modulator," IEEE J. Solid-State Circuits, vol. 36, no. 12, pp. 1879-1886, Dec. 2001.

[19] R. Schreier, "Quadrature mismatch shaping," in Proc. IEEE ISCAS, vol. 4, 2002, pp. 675-678.

[20] Y. Palaskas, Y. Tsividis, V. Prodanov, and V. Boccuzzi, “A "divide and conquer" technique for implementing wide dynamic range continuoustime filters," IEEE J. Solid-State Circuits, vol. 39, no. 2, pp. 297-307, Feb. 2004.

[21] Part 11: Wireless LAN Medium Access Control (MAC) and Physical Layer Specifications, IEEE Std., 2000.

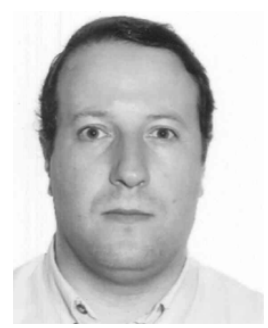

Jesus Arias (M'04) received the Licenciado en Física degree in 1989 and the Ph.D. degree in sciences (physics) in 1995, both from the University of Valladolid, Spain.

In 1989 he joined the Departamento de Electricidad y Electrónica, University of Valladolid, where he is now a Professor in electronics. He has been working in electrical characterization of semiconductor materials and devices, including computer simulation of semiconductor processing. His current research interests are the design of analog/digital converters for communication systems and the design of analog circuits for low-power, low-voltage applications.

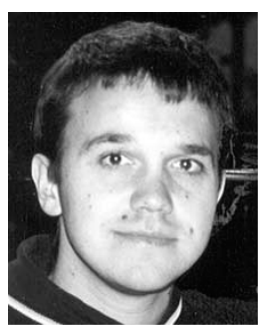

Peter Kiss (S'99-M'00) received the Engineer's, M.S. and Ph.D. degrees, all in electrical engineering, from the Technical University of Timişoara, Romania, in 1994, 1995, and 2000, respectively.

From 1998 to 2000 he was a research scholar at Oregon State University, Corvallis, working on correction techniques for fast and accurate delta-sigma converters. Since 2001, he has been with Agere Systems (formerly part of Bell Laboratories, Lucent Technologies) in Murray Hill, NJ, and Allentown, PA, dealing with data converters and analog filters for wireless systems. His past work involved adaptive fuzzy systems and image processing.

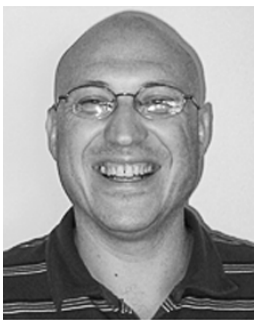

Vladimir Prodanov received M.S. and Ph.D. degrees in electrical engineering from the State University of New York, Stony Brook, in 1995 and 1997, respectively.

He was with Bell Laboratories, Silicon Circuit Research for four years and with Agere Systems (formerly Lucent Microelectronics) for three years as a Member and Senior Member of Technical Staff. Currently, he works as an individual technical consultant. His main interests are in the area of analog, RF and mixed-mode IC design as well as RF systems. He has designed various circuits for Bluetooth and IEEE $802.11 \mathrm{a} / \mathrm{b} / \mathrm{g}$ wireless standards. He has also worked on low-voltage CMOS circuits, high-voltage CMOS I/O buffers, biasing of high-power RF transistors and others. He has received eight patents and has contributed to some two dozen conference and journal publications. He has taught analog/RF IC design courses at SUNY, Stony Brook, and most recently at Columbia University, New York.

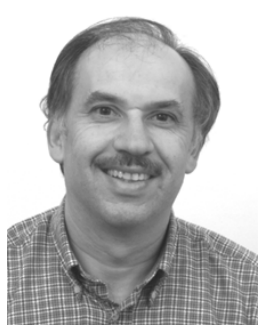

Vito Boccuzzi received the B.S. degree in electrical engineering from the New Jersey Institute of Technology, Newark, in 1980, and the M.S. degree from Adelphi University, Garden City, NY, in 1990.

From 1980 to 1996, he worked at AIL Systems (Airborne Instrumentation Laboratory) N.Y. in the Instrumentation division as an Analog Designer for synthesized signal generators and spectrum analyzers. He then moved to the Radar division where he was involved in the design of receivers for airborne doppler radars. In 1996, he joined Lucent Technologies/Bell Laboratories as a Member of Technical Staff, and then joined Agere Systems. With members of Communication Circuit Research, he designed and tested the prototype analog IC for wireless applications. Currently, he is with Analog Devices, Somerset, NJ, working on a direct-conversion receiver for satellite radio applications.

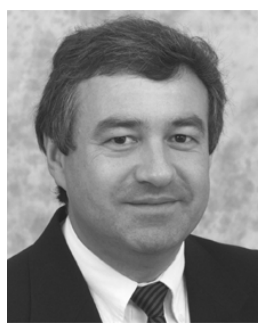

Mihai Banu (S'80-M'82-SM'96-F'02) received the B.S., M.S., and Ph.D. degrees in electrical engineering from Columbia University, New York, NY, in 1979, 1980, and 1984, respectively. His doctoral studies were sponsored by AT\&T Bell Laboratories, Murray Hill, NJ, where he worked during the summers of 1980-1983.

In 1984, he joined Bell Labs as a permanent employee and in 1996 he was appointed Head of the Silicon Circuits Research Department. This organization, now part of Lucent Technologies Bell Labs, was responsible for advanced work in analog and RF circuit design and $\mathrm{Si}$ technology/device enhancements, including developing a best in class $\mathrm{SiGe}$ BiCMOS technology. In 2001, his organization joined Agere Systems, a Lucent Technologies spin-off, and focused on wireless LAN work. In 2003, he was appointed Director, RF/Analog ICs in Agere Systems RF Power organization, where he worked on novel power amplifier architectures and support circuits. After retiring from Agere Systems in 2005, he has been an independent consultant with MHI Consulting, LLC, which he founded. His technical interests are in the fields of integrated circuit design, signal processing, and RF systems. Among his accomplishments are the invention of MOSFET-C continuous-time filters (1987 IEEE Darlington Award), contributions to integrated operational amplifier design (1998 Lucent Technologies patent award), contributions to high-speed communication circuits design, novel RF IC architectures for wireless, experimental work in multi-gigahertz wideband HBT circuits, and pioneering work in burst-mode clock recovery circuits. He holds several U.S. and international patents and has published many technical papers. He has been an Adjunct Professor at Columbia University and has taught several short courses in international conferences and workshops. He is a member of Eta Kappa $\mathrm{Nu}$ and Sigma $\mathrm{Xi}$ and has been an editor for IEEE CAS Society publications. 


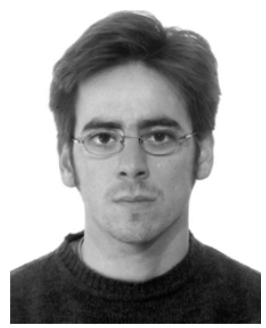

David Bisbal received the Telecommunication Engineer degree from the University of Valladolid, Spain, in 2002, where he is currently working toward the Ph.D. degree. His doctoral research focuses on the design of CMOS high-speed delta-sigma A/D converters.

Since 2002, he has worked as a Junior Lecturer in the Department of Electronics at University of Valladolid.

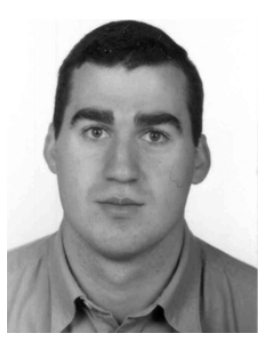

Jacinto San Pablo received the Electronics Engineering degree from the University of Valladolid, Spain, in 1999. He is currently working toward the Ph.D. degree at the University of Valladolid. His doctoral work focuses on the design of continuous-time sigma-delta modulators.

From 2000 to 2002, he worked at Silicon and Software Systems, Dublin, Ireland. In 2003, he joined the Departamento de Electricidad y Electrónica, University of Valladolid, Spain, where he is an Assistant Professor.

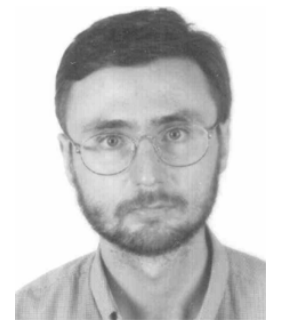

Luis Quintanilla received the Licenciado en Física degree in 1991 and the Ph.D. degree in sciences (physics) in 1993, both from the University of Valladolid, Spain.

He joined the Departamento de Electricidad y Electrónica, University of Valladolid, in 1993 where he is now a Professor in electronics. $\mathrm{He}$ has been working in electrical characterization of semiconductor materials and devices, and his current research interest is the design of analog circuits for low-power, low-voltage applications and high-speed data converters.

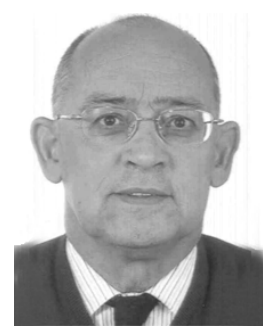

Juan Barbolla received the Licenciado en Física degree in 1969 from the University of Valladolid, Spain, and the Ph.D. degree in sciences (physics) in 1976 from the University Paul Sabatier, Toulouse, France, and from the University of Valladolid, Spain.

He joined the Departamento de Electricidad y Electrónica, University of Valladolid, in 1983, where he is now a Full Professor in electronics and head of the Department. His research includes electrical characterization of semiconductor devices, computer simulations of semiconductor processing, and he is also interested in the design of analog circuits. 